\title{
Obanomics: desequilibrios políticos y económicos de seis años
}

Rómulo Daniel Montes de Oca Zárate*

Durante la presente administración demócrata, iniciada el 20 de enero de 2009, por el cuadragésimo cuarto presidente de los Estados Unidos de América, Barack Obama, la economía estadounidense ha experimentado diversos embates, los cuales han significado un gran reto para distintas instituciones tanto privadas como gubernamentales. La gráfica 1 muestra una tendencia a la baja del producto norteamericano la cual no ha mejorado luego de la crisis financiera de 2008; por su parte la inflación se ha mantenido en torno al blanco numérico de la Reserva Federal (Fed) de $2 \%$ a partir de 2010 .

Bajo tal panorama, este artículo buscará identificar las tendencias políticas y económicas durante la administración demócrata. La primera sección analizará los resultados de las elecciones intermedias, lo que permite ver la aceptación que las políticas del presidente han tenido en Estados Unidos. En la segunda sección se analizarán variables de la cuenta pública como la deuda y el déficit federales, y algún otro concepto, con lo que se atisbará el enfoque seguido por la administración.

\section{Elecciones intermedias}

Las elecciones entre periodos federales son un indicador potente de la aceptación que ha logrado el partido en turno dentro de la administración en general, pero con especial énfasis respecto a la cabeza del poder ejecutivo. Durante la gestión de Obama el legislativo se ha visto como un contrapeso no dominante, debido a que a pesar de una mayoría republicana

\section{Gráfica I}

EUA: producto real e inflación, 2004-2013

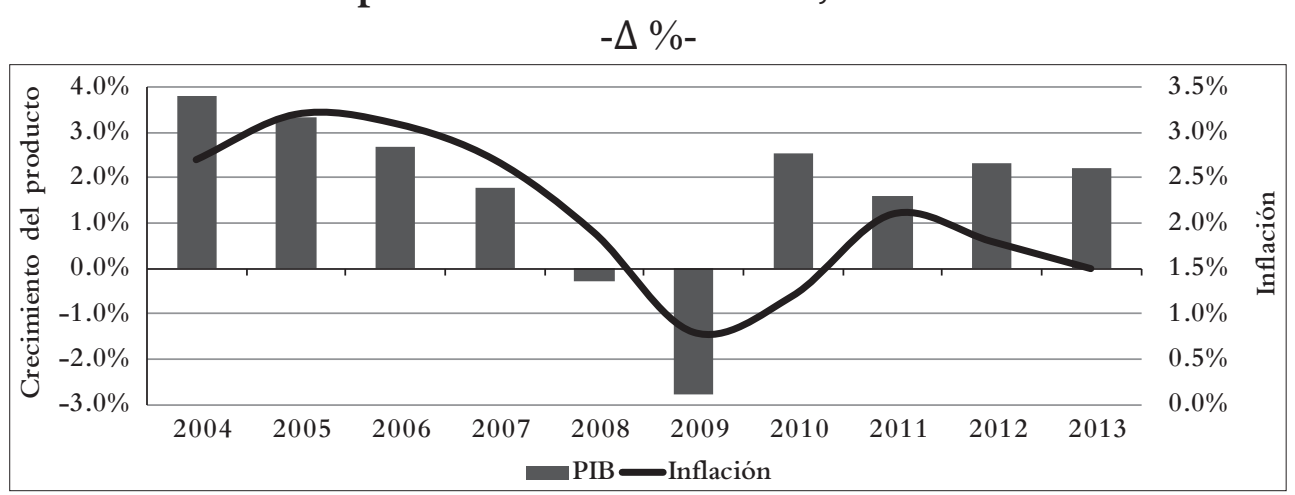

Fuente: elaboración propia con base en Bureau of Economic Analysis, 2014.

\footnotetext{
* Miembro del Seminario de Credibilidad Macroeconómica, FE-UnAM.
} 


\section{Gráfica 2}

EUA: rebalanceo del congreso, 2012-2014

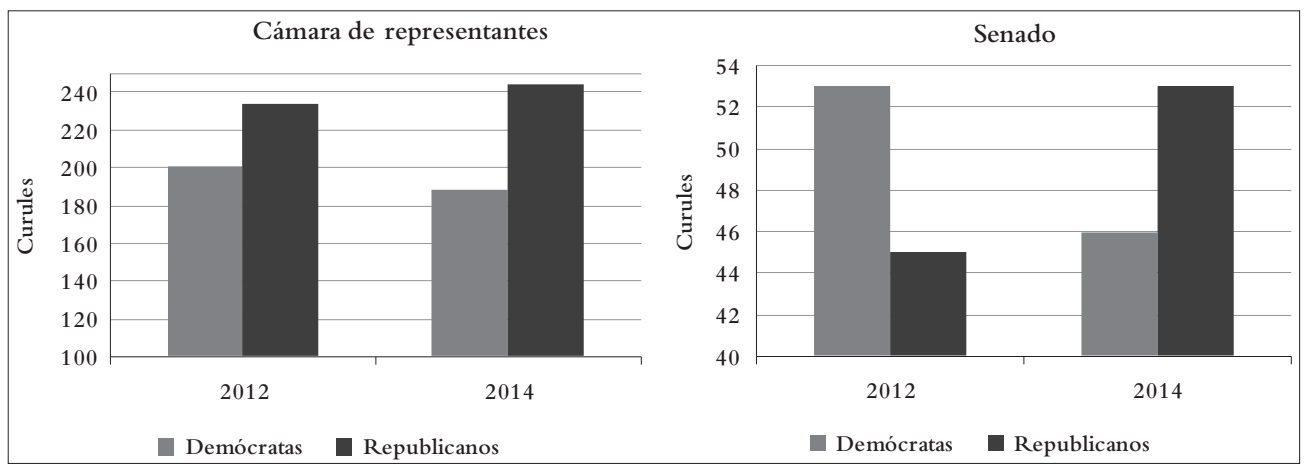

Fuente: elaboración propia con base en datos del Wall Street Journal.

dentro de la cámara de representantes, el senado ha sido ocupado mayormente por candidatos demócratas. Diversas polémicas dentro de la administración de Obama llevaron a los principales diarios estadounidenses, así como a diversos centros estadísticos, a pronosticar un reajuste de poder en los órganos legislativos durante las elecciones del pasado 4 de noviembre.
Como se aprecia en la gráfica 2 las elecciones intermedias durante el primer término de la presidencia de Obama, el partido demócrata logró dominar el senado en cuanto a curules respecta; para las elecciones de 2014 el peso de los demócratas fue arrasado por completo por el partido republicano. A pesar de que la composición de la cámara de representantes se mantuvo estable, la gráfica

\section{Gráfica 3}

\section{Aceptación política del ejecutivo y legislativo, 2014}

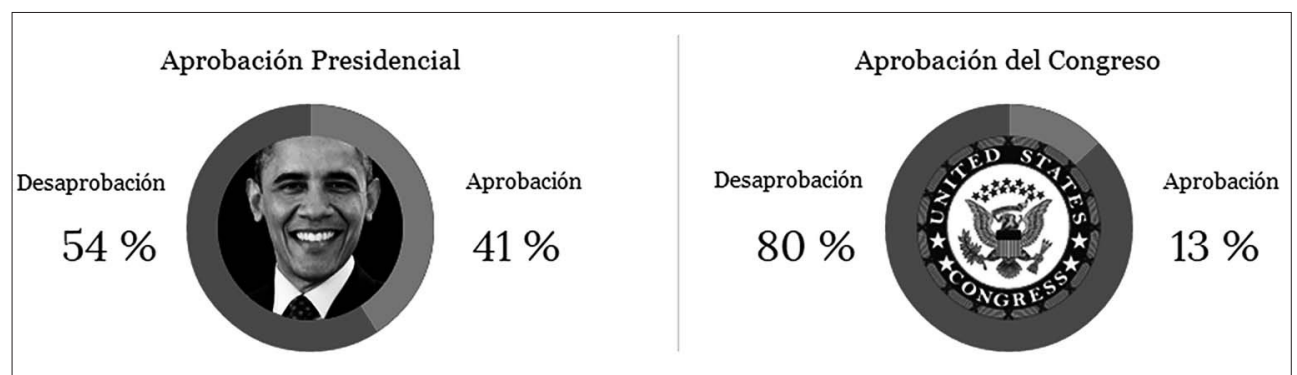

Fuente: elaboración propia con base en datos de Fox News. 
también muestra un ligero aumento de votaciones en favor del partido republicano, que no ha ocupado la Casa Blanca desde la conclusión de la presidencia de W. Bush. La gráfica 3 muestra la aprobación tanto ejecutiva como legislativa con la nueva composición de las cámaras; es claro que el presidente ha perdido poco a poco simpatía entre el electorado, mientras que el congreso mantiene una desaprobación generalizada debido a la falta de resolución en la toma de decisiones.

Finalmente la gráfica cuatro muestra la recomposición de los asientos ocupados por los partidos en ambas cámaras legislativas. Teniendo en cuenta la clara redistribución hacia la preponderancia del partido republicano, es posible mostrar que el electorado norteamericano no está convencido de las políticas aplicadas por el partido demócrata, que actualmente controla la presidencia. La respuesta de los republicanos a la pérdida en las urnas, se explica por una polarización política lo cual hace de la gobernanza una tarea difícil, la respuesta poco satisfactoria lleva a otras posibles conclusiones.

¿Es acaso la situación económica de una precaria recuperación con alto desempleo y de las malas condiciones de vida los que han llevado a la ciudadanía americana a virar su voto al partido conservador? El siguiente apartado buscará observar el comportamiento económico de la administración de Obama para así evaluar si la recuperación, que de acuerdo al ejecutivo ha seguido un camino aceptable, en verdad logra impulsar el espíritu de desarrollo y crecimiento

\section{Gráfica 4}

EUA: resultados electorales 2014

-elecciones intermedias-

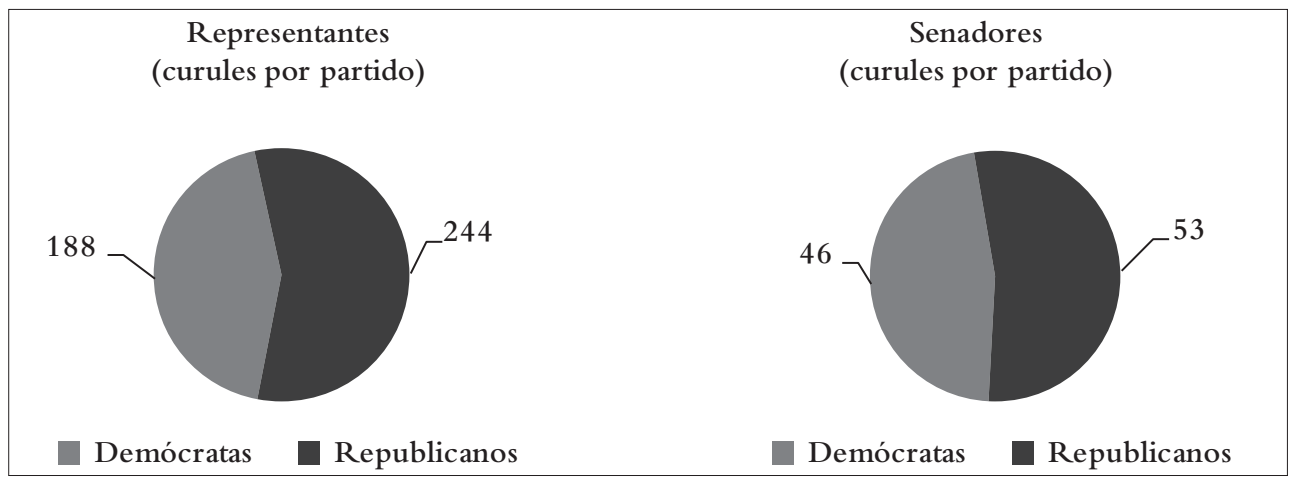

Fuente: elaboración propia con base en datos del Wall Street Journal. 
Economía Informa núm. 389 noviembre - diciembre • 2014 = " " " "

que caracterizó el siglo pasado de los Estados Unidos.

\section{Política fiscal ineficiente}

La más reciente administración de la Casa Blanca se ha enfrentado a una de las recesiones más severas dentro de la economía estadounidense, la cual ha tenido enormes impactos en el seno de uno de los estratos más importantes de su economía, el sector financiero. La principal respuesta de los asesores económicos de Obama fue el impulso de la economía vía un incremento desmedido en el gasto público, llevando a niveles de endeudamiento histórico. Pese a que la recesión fue fechada por el NBER de diciembre de 2007 a junio de 2009, definiendo un periodo que justificaría un aumento en la deuda con motivo de ajuste económico vía política anticíclica, una vez comenzada la administración de Obama el incremento de la deuda federal continuó escalando hasta alcanzar niveles históricos.

El crecimiento de déficit presupuestario y la deuda pública (como porcentaje del PIB) durante el segundo término de la administración republicana de W. Bush y lo que va de la administración demócrata de Obama son presentados dentro de la gráfica 5 . Claramente el déficit presupuestal incrementó en respuesta ante la recesión

\section{Gráfica 5}

EUA: déficit* y deuda gubernamentales, 2004-2014

-como \% del PIB-

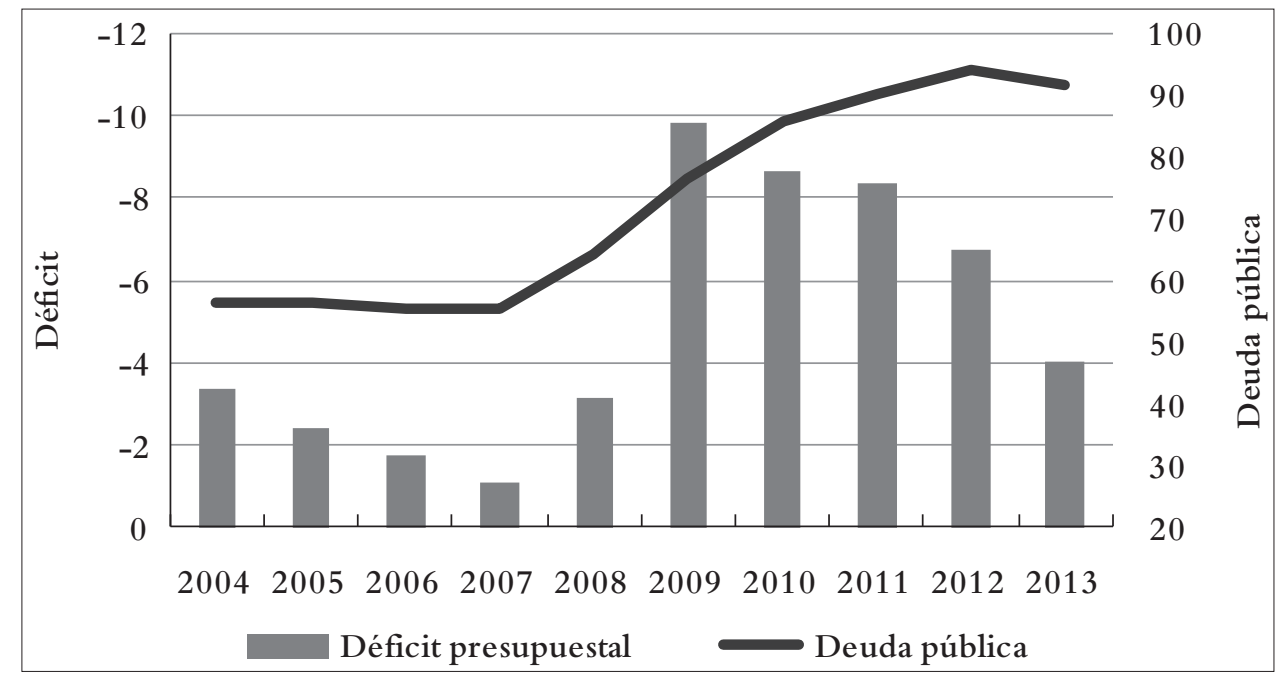

*Escala invertida.

Fuente: elaboración propia con base en datos de BEA y FRED. 
económica de 2007 para después de 2009 disminuir gradualmente a los niveles precedentes al fenómeno; a pesar de que esta variable tiende a disminuir la deuda ha continuado con un incremento exponencial a lo largo de la administración de Obama.

Si se busca analizar los efectos de la política económica contracíclica, es necesario observar qué comportamiento ha seguido el desempleo con el incremento expansivo de la deuda pública. La gráfica 6 muestra que el detrimento económico que significó tasas de crecimiento negativas durante 2008 y 2009 , comenzó a recobrar dinamismo a partir de 2010 , pero la tasa de desempleo no ha logrado los niveles previos a la recesión. El claro retorno del producto a su nivel de partida, no se refleja en la tasa de desempleo. Para mostrar de mejor manera este efecto se optó por igualar los valores iniciales de ambas variables a cero, normalizando el resto de la serie para evaluar su desempeño. A partir de esta técnica se encuentra una evidente brecha entre el producto y el alto desempleo, situación mejor conocida como jobless recovery (véase flecha en el gráfico).

Teniendo este panorama en cuenta es posible sostener que el aumento excesivo de la deuda no ha sido benéfico para la economía norteamericana en su conjunto; en este sentido el mal desempeño económico en el sector laboral se ha aunado al desequilibrio financiero (mayor porcentaje de deuda) vulnerando la estructura interna

\section{Gráfica 6}

EUA: producto real y desempleo, 2004-2013

$-\Delta \%$ con los valores iniciales igualados a cero-

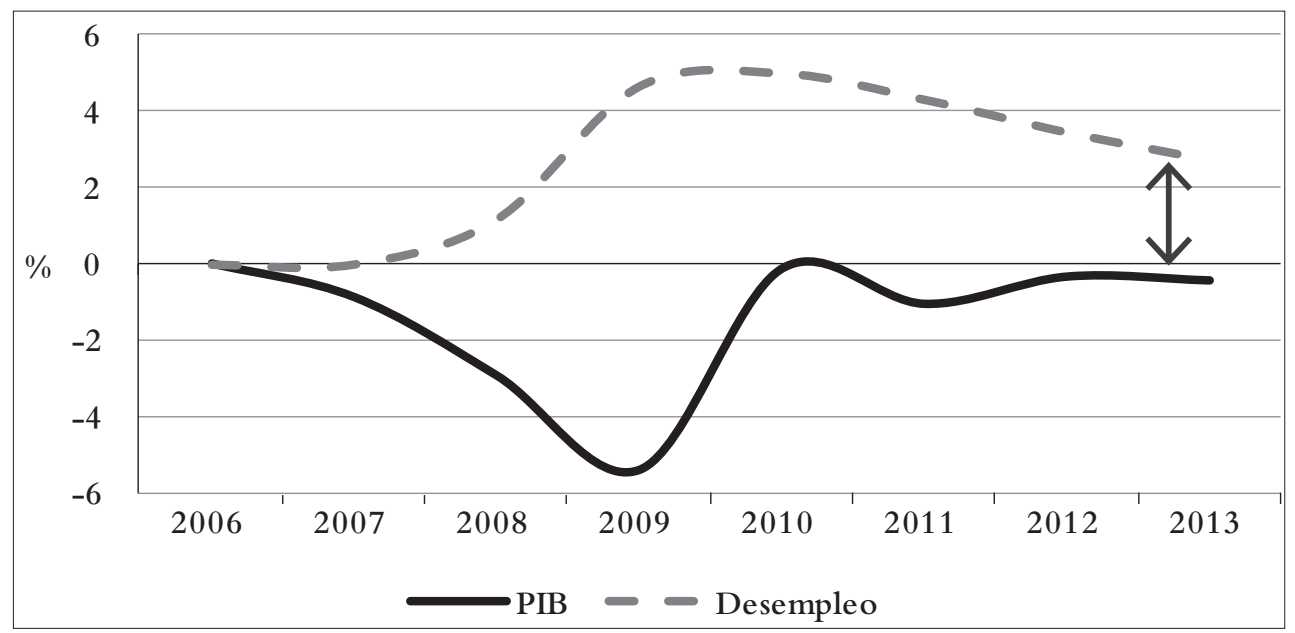

Fuente: elaboración propia con base en datos de la BEA y del BLS. 
del país, la cual se ve cada vez más comprometida debido a la incertidumbre en torno al panorama económico futuro,

\section{Conclusiones}

Las más recientes elecciones en los Estados Unidos han intensificado la polarización existente entre los dos partidos políticos; bajo esta polaridad es posible prever diversos choques entre la presidencia demócrata y la legislatura republicana, de los cuales algunos ya se han hecho presentes con el reciente reacomodo del gabinete y con la decisión unilateral del ejecutivo en materia migratoria.

La disputa entre los más altos órganos gubernamentales de Estados Unidos se suma a dos hechos planteados a partir de la política económica de Obama. En primer lugar, dado lo visto en la segunda sección se encuentra que la política económica expansiva no ha logrado disminuir los problemas de la economía norteamericana; a pesar de una recuperación en los niveles de crecimiento, la calidad de vida de los estadounidenses ha disminuido drásticamente, lo que representa un malestar social generalizado.

En segundo lugar, los problemas sociales que ha traído consigo la actual recesión, así como el estancamiento paulatino de la economía familiar en los Estados Unidos, han generado un cambio radical dentro de las expectativas de los agentes económicos. La manera más simple de observar el desacuerdo social con el gobierno federal es la supresión del voto de confianza hacia el partido del ejecutivo en las elecciones intermedias. La pregunta que sólo los agentes y las condiciones económicas podrán responder en los próximos dos años es: ¿será el ejecutivo capaz de restaurar la economía y la confianza del electorado para la siguiente contienda de la Casa Blanca o el partido demócrata estará condenado a una nueva desaprobación a causa de las decisiones arbitrarias del presidente Obama? 


\section{Referencias}

Bureau of Economic Analysis (2014), National, interactive tables, GDP, Federal Debt and Expenditure.

Bureau of Labor Statistics (2014), Databases, tables \& calculators, unemployment.

Federal Reserve Economic Data (2014), Graph observations, Economic research division.

Fox News (2014), Politics, Midterm elections coverage.

James, Taranto (2014), "Midterm elections: how democrats spin losing" Wall Street Journal, noviembre 4.

Martin, Jurek (2014), "US midterm elections 2014: Republicans go into Cruz control" Financial Times, noviembre 6.

Patton, Mike (2014), “The Fed, monetary expansion, and inflation” Forbes, junio 30.

National Bureau of Economic Research (2014), Business cycle date. 